International Journal of Biological Sciences

ISSN 1449-2288 www.biolsci.org 20084 (1): 8-14

Review

CIvyspring International Publisher. All rights reserved

\title{
Primary antioxidant free radical scavenging and redox signaling pathways in higher plant cells
}

\author{
Hong-Bo Shao 1, 2, 3, Li-Ye Chu ${ }^{3}$, Zhao-Hua Lu 1, 4, Cong-Min Kang ${ }^{3}$ \\ 1. Binzhou University, Binzhou 256603, China \\ 2. Institute of Soil and Water Conservation, Chinese Academy of Sciences, Yangling 712100, China \\ 3. Institute for Life Sciences, Qingdao University of Science \& Technology (QUST), Qingdao 266042, China \\ 4. Institute of Restoration Ecology, China University of Mining \& Technology, Beijing100083, China
}

Correspondence to: Dr. Professor Shao Hong-Bo and Lu Zhao-Hua, Email: shaohongbo@qust.edu.cn; lu-zhh@263.net. Dr. Professor Shao Hong-Bo, Institute for Life Sciences, Qingdao University of Science \& Technology, Qingdao 266042, China. Tel.: +86-532-84023984; Fax: $+86-532-84023271$.

Received: 2007.10.14; Accepted: 2007.12.04; Published: 2007.12.07

Antioxidants in plant cells mainly include glutathione, ascorbate, tocopherol, proline, betaine and others, which are also information-rich redox buffers and important redox signaling components that interact with cellular compartments. As an unfortunate consequence of aerobic life for higher plants, reactive oxygen species (ROS) are formed by partial reduction of molecular oxygen. The above enzymatic and non-enzymatic antioxidants in higher plant cells can protect their cells from oxidative damage by scavenging ROS. In addition to crucial roles in defense system and as enzyme cofactors, antioxidants influence higher plant growth and development by modifying processes from miotosis and cell elongation to senescence and death. Most importantly, they provide essential information on cellular redox state, and regulate gene expression associated with biotic and abiotic stress responses to optimize defense and survival. An overview of the literature is presented in terms of primary antioxidant free radical scavenging and redox signaling in plant cells. Special attention is given to ROS and ROS-anioxidant interaction as a metabolic interface for different types of signals derived from metabolisms and from the changing environment. This interaction regulates the appropriate induction of acclimation processes or execution of cell death programs, which are the two essential directions for higher plant cells.

Key words: Plant cells; Antioxidant; Reactive oxygen species (ROS); ROS-antioxidant interacting interface; Soil-plant interface; Signaling

\section{Introduction}

Higher plants survive in a constantly fluctuating environment, which has driven the evolution of a highly flexible metabolism and growth (basic growth curve of slow-fast-slow) and development necessary for their sessile lifestyle [1-3, 17-19]. The cellular state is defined as the total active level that regulates cells to adapt to their surroundings in a narrow sense $[4,10$, 13-16]. Redox state is the important part of the above cellular state and one of the most important indicators for evaluating the above state [4, 9, 14-16]. Primary intracellular plant antioxidants expression (or levels or location) are closely related to their metabolic state and is responding to constantly fluctuating environment [5-9]. Practically, this phenomenon is also quite similar to animals in most cases. Because higher plants are sessile, they develop into a series of pathways at different levels that combat with environmental stress, which produces more ROS [10-16]. These pathways include the phtorespiratory pathway, enzymatic and non-enzymatic pathways, corresponding respon- sive-gene regulation and anatomical ways [17-21]. General stress includes drought, salinity, low temperature, UV-B and others. Currently, in contrast to the situation in the natural world, the detailed dissection of the regulatory networks that govern higher plants' responses to abiotic insults and their biotic stress aspects have been studied almost exclusively in controlled environments where a single challenge has been applied [4-12, 19-21]. Plant cell metabolism must be highly regulated in order to allow effective integration of a diverse spectrum of biosynthetic pathways that are reductive in nature [13-15, 61-64], in which the regulation does not completely avoid photodynamic or reductive activation of molecular oxygen to produce ROS, particularly superoxide, $\mathrm{H}_{2} \mathrm{O}_{2}$ and singlet oxygen [15-17]. However, in many cases, the production of ROS is genetically programmed, induced during the course of development and by environmental fluctuations, and has complex downstream effects on both primary and secondary metabolism [18-20, 59-64]. Higher plant cells produce ROS, particularly superoxide and $\mathrm{H}_{2} \mathrm{O}_{2}$ and, as second messengers in many 
processes associated with plant growth and development [21-26, 62]. Moreover, one of the major ways in which higher plants transmit information concerning changes in the changing environment is via the production of bursts of superoxide at the plasma membrane $[27,28]$.

Situations which provoke enhanced ROS production have in the past been categorized under the heading of oxidative stress, which in itself is a negative term implying a harmful process, when in fact it is probably in many cases quite the opposite, enhanced oxidation being an essential component of the repertoire of signals that higher plants use to make appropriate adjustments of gene expression and cell structure in response to environmental and developmental cues [29, 30, 61-64]. Rather than involving simple signaling cassettes, emerging concepts suggest that the relationship between metabolism and redox state is complex and subtle [31,63]. Related functioning processes for main antioxidants and redox signaling in plant cells were summarized in Figure 1. This article covered several important aspects of antioxidants and redox signaling in higher plants, including biological roles of main antioxidants, ROS and redox signaling, Antioxidants and redox sensing mechanisms, and A network for redox signaling.

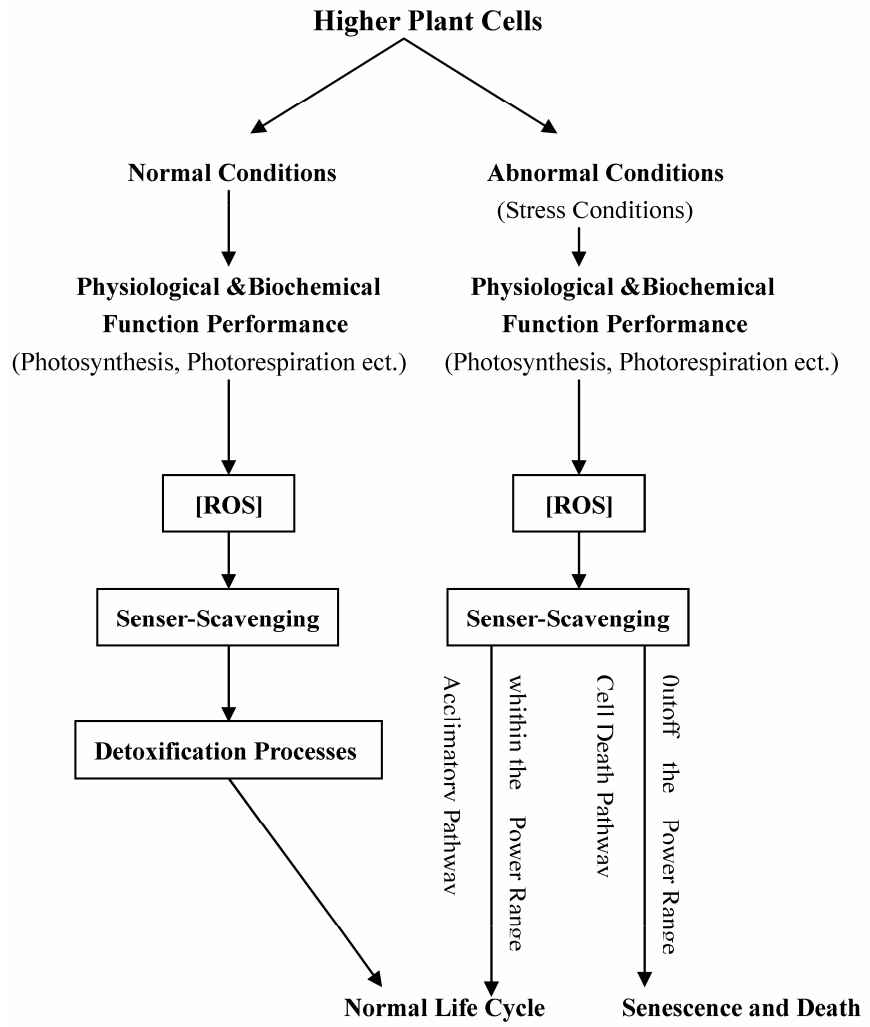

Figure 1 Related processes for main antioxidants and redox signaling in plant cells $[43,53,57]$

\section{Biological Roles of Main Antioxidants in Plant Cells}

\section{1. $\alpha$ - tocopherol}

a-tocopherol, found in green parts of plants scavenges lipid peroxy radicals through the concerted action of other antioxidants [31, 45]. Further, tocopherols were also known to protect lipids and other membrane components by physically quenching and chemically reacting with $\mathrm{O}_{2}$ in chloroplasts, thus protecting the structure and function of PSII $[32,46]$. Researchers reported a two-fold increase in a-tocopherol in turf grass under water stress [33, 47].

In plant cells, the most important reducing substrate for $\mathrm{H}_{2} \mathrm{O}_{2}$ removal is ascorbic acid [38, 39, 62]. A direct protective role for ascorbic acid has also been demonstrated in rice, where partial protection against damage caused by a release from flooding conditions was provided by the prior addition of ascorbic acid [40]. a -Tocopherols (vitamin E) are lipophilic antioxidants synthesized by all plants. a -Tocopherols interact with the polyunsaturated acyl groups of lipids, stabilize membranes, and scavenge and quench various reactive oxygen species (ROS) and lipid soluble byproducts of oxidative stress [41,42].

Singlet oxygen quenching by tocopherols is highly efficient, and it is estimated that a single a-tocopherol molecule can neutralize up to 120 singlet oxygen molecules in vitro before being degraded [43]. Because of their chromanol ring structure, tocopherols are capable of donating a single electron to form the resonance - stabilized tocopheroxyl radical [44]. a tocopherols also function as recyclable chain reaction terminators of polyunsaturated fatty acid (PUFA) radicals generated by lipid oxidation [45]. a-tocopherols scavenge lipid peroxy radicals and yield a tocopheroxyl radical that can be recycled back to the corresponding a - tocopherol by reacting with ascorbate or other antioxidants [46]. a - tocopherols are major lipid soluble antioxidant present in the PUFA-enriched membranes of chloroplasts and are proposed to be an essential component of the plastid antioxidant network. The most attributed function of tocopherols is their involvement in various mechanisms in protecting PUFAs from oxidation [47]. ROS generated as by-products of photosynthesis and metabolism are potential sources of lipid peroxidation in plant cells. a - tocopherol levels increase in photosynthetic plant tissues in response to a variety of abiotic stresses [48]. a-tocopherols scavenge and quench various ROS and lipid oxidation products, stabilize membranes, and modulate signal transduction $[48,49]$.

Synthesis of low-molecular-weight antioxidants, 
such as a -tocopherol, has been reported in drought-stressed plants [49]. Oxidative stress activates the expression of genes responsible for the synthesis of tocopherols in higher plants $[49,50]$. Antioxidants including a-tocopherol and ascorbic acid have been reported to increase following triazole treatment in tomato and these may have a role in protecting membranes from oxidative damage, thus contributing to chilling tolerance [51]. Triazole increases the levels of antioxidants and antioxidant enzymes in wheat [52]. Photosynthetic apparatus and membrane could be affected by water stress. Alpha-tocopherol is a lipid-soluble antioxidant associated with biological membrane of cells, especially the membrane of photosynthetic apparatus [8, 10, 14, 21]. Research has shown that water deficiency may result in an increase of tocopherol concentration in plant tissues [50,51]. Some evidence implied that tocopherol content of soybean leaves was increased as the amount of rainfall decreased [52]. This is consistent with the reports of [53], which showed that subjecting spinach to water deficit increased the content of -tocopherol in the leaves. Based on the studies of 10 different grass species under water stress, the researcher found that drought stress led to an increase of 1 to 3 -fold of -tocopherol concentration in 9 out of 10 species $[53,60]$. They pointed out that the species with a high tolerance of stress are defended through tocopherol. Moreover, highly significant correlations were observed between stress tolerance and -tocopherol concentration (the precursor of a-tocopherol; Spearmans rank correlation coefficient $r=0.731$ ).

\subsection{Ascorbic acid (AA)}

AA is one of the most extensively studied anti-oxidants and has been detected in the majority of plant cell types, organelles and apoplast [34,35]. AA is synthesized in the mitochondria and is transported to the other cell components through a proton - electrochemical gradient or through facilitated diffusion. Further, AA also has been implicated in regulation of cell elongation [36, 49]. In the ascorbate-glutathione cycle, two molecules of AA are utilized by APX to reduce $\mathrm{H}_{2} \mathrm{O}_{2}$ to water with concomitant generation of monodehydroascorbate. Monodehydro-ascorbate is a radical with a short life time and can dispropotionate into dehydroascorbate and ascorbic acid. The electron donor is usually NADPH and catalyzed by monodehydro-ascorbate reductase or ferredoxin in water-water cycle in the chloroplasts [37].

A continuous oxidative assault on plants during drought stress has led to the presence of an arsenal enzymatic and non enzymatic plant antioxidant defenses to counter the phenomenon of oxidative stress in plants [54]. AA is an important antioxidant, which reacts not only with $\mathrm{H}_{2} \mathrm{O}_{2}$ but also with $\mathrm{O}_{2}{ }^{-}, \mathrm{OH}$ and lipid hydroperoxidases $[46,55]$. AA is water soluble and also has an additional role in protecting or regenerating oxidized carotenoids or tocopherols $[55,56]$. Water stress resulted in significant increase in antioxidant AA concentration in turf grass [57].

AA showed a reduction under drought stress in maize and wheat, suggesting its vital involvement in deciding the oxidative response [58]. Some reports reported a decrease in the level of antioxidants including AA with increase in stress intensity in wheat. AA can also directly scavenge $1 \mathrm{O}_{2}, \mathrm{O}_{-2}$ and $\bullet \mathrm{OH}$ and regenerate tocopherol from tocopheroxyl radicals, thus providing membrane protection $[58,59]$. AA also acts as a co-factor of violaxanthin de-epoxidase, thus sustaining dissipation of excess excitation energy $[59,60]$. Antioxidants such as AA and glutathione are involved in the neutralization of secondary products of ROS reactions $[45,61]$.

Ascorbate (vitamin C) occurs in all plant tissues, usually being higher in photosynthetic cells and meristems (and some fruits). About 30 to $40 \%$ of the total ascorbate is in the chloroplast and stromal concentrations as high as $50 \mathrm{mM}$ have been reported [62]. It is highest in the mature leaf, where the chloroplasts are fully developed and the chlorophyll levels are highest. Although it has been determined that D-glucose is the precursor of L-AA, the synthetic pathway has not been totally understood $[61,63]$. AA has effects on many physiological processes including the regulation of growth, differentiation and metabolism of plants. A fundamental role of AA in the plant defense system is to protect metabolic processes against $\mathrm{H}_{2} \mathrm{O}_{2}$ and other toxic derivatives of oxygen. Acting essentially as a reductant and reacting with and scavenging many types of free radicals, AA reacts non-enzymatically with superoxide, hydrogen peroxide, and singlet oxygen. It can react indirectly by regenerating -tocopherol or in the synthesis of zeaxanthin in the xanthophyll cycle. Therefore, AA influences many enzyme activities, and minimizes the damage caused by oxidative process through synergic function with other antioxidants [60-63].

\subsection{Reduced glutathione}

Glutathione is a tripeptide (a-glutamyl cysteinylglycine), which has been detected virtually in all cell compartments such as cytosol, chloroplasts, endoplasmic reticulum, vacuoles and mitochondria [64]. Glutathione is the major source of non-protein thiols in most plant cells. The chemical reactivity of the thiol group of glutathione makes it particularly suitable to serve a broad range of biochemical functions in all organisms. The nucleophilic nature of the thiol group also is important in the formation of mercaptide bonds 
with metals and for reacting with selected electrophiles. This reactivity along with the relative stability and high water solubility of GSH makes it an ideal biochemical to protect plants against stress including oxidative stress, heavy metals and certain exogenous and endogenous organic chemicals $[63,64]$.

Glutathione takes part in the control of $\mathrm{H}_{2} \mathrm{O}_{2}$ levels $[56,63]$. The change in the ratio of its reduced (GSH) to oxidized (GSSG) form during the degradation of $\mathrm{H}_{2} \mathrm{O}_{2}$ is important in certain redox signaling pathways [64]. It has been suggested that the GSH/GSSG ratio, indicative of the cellular redox balance, may be involved in ROS perception [63, 64]. Reduced glutathione (GSH) acts as an antioxidant and is involved directly in the reduction of most active oxygen radicals generated due to stress. There was a report reporting that glutathione, an antioxidant helped to withstand oxidative stress in transgenic lines of tobacco $[39,63]$.

\section{ROS and Redox Signaling}

Higher plants, as other aerobic organisms, require oxygen for the efficient production of energy [40]. During the reduction of $\mathrm{O}_{2}$ to $\mathrm{H}_{2} \mathrm{O}$, reactive oxygen species (ROS), namely superoxide radical $\left(\mathrm{O}_{2}{ }^{\bullet}\right)$, hydrogen peroxide $\left(\mathrm{H}_{2} \mathrm{O}_{2}\right)$, and hydroxyl radical $\left(\mathrm{OH}^{\bullet}\right.$ ) can be formed [41, 42]. Most cellular compartments in higher plants have the potential to become a source of ROS. Environmental stresses that limit $\mathrm{CO}_{2}$ fixation, such as drought and salt stress, ozone and high or low temperatures, reduce the $\mathrm{NADP}^{+}$regeneration by the Calvin cycle, consequently, the photosynthetic electron transport chain is over-reduced, producing superoxide radicals and singlet oxygen in the chloroplasts [43-45, 56, 59]. To prevent over-reduction of the electron transport chain under conditions that limit $\mathrm{CO}_{2}$ fixation, higher plants evolved the photorespiratory pathway to regenerate NADP+ $[47,49]$. As part of the photorespiratory pathway, $\mathrm{H}_{2} \mathrm{O}_{2}$ is produced in the peroxisomes, where it can also be formed during the catabolism of lipids as a by-product of $\beta$-oxidation of fatty acids [50, 62].

Because of the highly cytotoxic and reactive nature of ROS, their accumulation must be under tight control. Higher plants possess very efficient enzymatic and non-enzymatic antioxidant defence systems that allow scavenging of ROS and protection of plant cells from oxidative damage [51-53, 60-62]. The distinct subcellular localization and biochemical properties of antioxidant enzymes, their differential inducibility at the enzyme and gene expression level and the plethora of non-enzymatic scavengers render the antioxidant systems a very versatile and flexible unit that can control ROS accumulation temporally and spatially [54,
61-64]. The above controlled modulation of ROS levels is significant in the light of the recent evidence for a signaling capacity of ROS [55, 60-64].

Higher plants can sense, transduce, and translate the ROS signals into appropriate cellular responses, the process of which requires the presence of redox-sensitive proteins that can undergo reversible oxidation/reduction and may switch 'on' and 'off 'depending upon the cellular redox state [56, 61]. ROS can oxidize the redox-sensitive proteins directly or indirectly via the ubiquitous redox-sensitive molecules, such as glutathione (GSH)or thioredoxins, which control the cellular redox state in higher plants $[57,63$, 65, 66]. Redox-sensitive metabolic enzymes may directly modulate corresponding cellular metabolism, whereas redox-sensitive signaling proteins execute their function via downstream signaling components, such as kinases, phosphatases, and transcription factors $[58,59,62]$. Currently, two molecular mechanisms of redox-sensitive regulation of protein function prevail in living organisms [23-25, 36, 42, 60-64]. Signaling mediated by ROS involves hetero-trimeric G-proteins [29] and protein phosphorylation regulated by specific MAP kinases and protein Tyr phosphatases [31, 63]. The biochemical and structural basis of kinase pathway activation by ROS remains to be established in higher plants, but thiol oxidation possibly plays a key role [37, 46, 62]. The best-characterized redox signal transduction system in higher plants is the stromal ferredoxin-thioredoxin system, which functions in the regulation of photosynthetic carbon metabolism. Signal transmission involves disulfide-thiol conversion in target enzymes and is probably achieved by a light-induced decrease in the thioredoxin redox potential from about $-0.26 \mathrm{~V}$ to about $-0.36 \mathrm{~V}[27,36,61]$. Thiol groups are likely important in other types of redox signal transduction, including ROS sensing by receptor kinases, such as ETR1 [48, 60]. ROS in higher plants must utilize and/or interfere with other signaling pathways or molecules, forming a signaling network [61, 64]. Increasing evidence shows that higher plant hormones are positioned downstream of the ROS signal. $\mathrm{H}_{2} \mathrm{O}_{2}$ induces accumulation of stress hormones, such as salicylic acid (SA) and ethylene [31, 36]. Tobacco plants exposed to ozone accumulate ABA and induction of the PDF1.21 gene by paraquat is impaired in Arabidopsis mutants insensitive to jasmonates (JA) and ethylene $[42,46]$. Higher plant hormones are not only located downstream of the ROS signal, but ROS themselves are also secondary messengers in many hormone signaling pathways [16, 19, 64-66]. No doubt, feedback or feedforward interactions may conceivably occur between different hormones and ROS [23, 28, 39, 57]. 


\section{Concluding Remarks}

Besides exacerbating cellular damage, ROS can act as ubiquitous signal molecules in higher plants. ROS are a central component in stress responses and the level of ROS determines the type of response. Antioxidants and ROS are an important interacting system with different functions in higher plants, which ensures themselves a highly flexible organism [35, 39, 58]. Redox signal transduction is a universal characteristic of aerobic life honed through evolution under natural and selecting pressure to balance information from metabolism and the changing environment [62-66]. Higher plant cells can be considered as a series of interconnecting compartments with different antioxidant buffering capacities determined by differences in synthesis, transport and/or degradation.There is a set of discrete locations where signaling is controlled (or buffered) independently in higher plant cells, which permits redox-sensitive signal transduction to occur in locations such as the appoplast, the thylakoid, and the endoplasmic reticulum, whereas other highly buffered spaces have a much higher threshold for ROS signals. Both oxidants and antioxidants fulfill signaling roles to provide information on higher plant health, particularly in terms of robustness for defense, using kinase-dependent and in-dependent pathways that are initiated by redox-sensitive receptors modulated by thiol status. Antioxidants are not passive bystanders in this crosstalk, but rather function as key signaling components that constitute a dynamic metabolic interface between higher plant cell stress perception and physiological responses. Increasing current data suggest that glutathione is a key arbiter of the intracellular redox potential, and ascorbate is particularly influential in setting threshold for apoplastic and cytoplasmic signaling [57, 60-66]. Differential antioxidant concentrations between compartments make antioxidant-driven vectorial signaling through processes such as ascorbate-driven electron transport or futile cycles. There can be no doubt that transgenic plants will be invaluable in assessing the precise role that main antioxidants and ROS play in the functional network that controls stress tolerance. Approximate redox potentials and intracellular concentrations of main redox couples in plant cells were illustrated in Table 1 [62, 63]. Although steps in the biosynthetic pathways resulting in antioxidant accumulation in higher plant cells have been primarily characterized at the physiological and molecular level, the full cast of participants involved in the complex regulation of their accumulation remains to be identified. This will require not only better understanding of the degradation and transport of antioxidants in higher plants, but also elucidation of the molecular events responsible for stress perception and stress-related signal transduction via wider scope of tested plants. Basic research leading to the characterization of tightly regulated stress-inducible promoters that are also responsive to appropriate tissue-specific regulation and endogenous developmental programmes is likely to be critical in improving the overall field performance of transgenic crops. The future will determine more precisely how ascorbate, glutathione, and tocopherol are involved in initiating and controlling redox signal transduction and how they trigger the gene expression of other related responses to optimize survival strategies. In addition, other problems are how antioxidants coordinate growth and development of higher plants in a constantly changing environment, how redox signaling is linked with hormonal regulation, nutrient status and redox potential of higher plants, and how their redox signaling is cooperated with inter-and intracellular signaling, transport capacity, developmental and environmental cues to maintain an appropriate dynamic homeostasis for stress tolerance and efficient survival.

Table 1 Approximate redox potentials and intracellular concentrations of main redox couples in plant cells (modified from Foyer and Noctor, 2005)

\begin{tabular}{|c|c|c|}
\hline Redox Couples a & $\begin{array}{l}\text { Redox Potentials } \\
(\mathrm{V})\end{array}$ & $\begin{array}{c}\text { Concentration Range ( } \\
\mu \mathrm{M})^{\mathrm{b}}\end{array}$ \\
\hline $\mathrm{O}_{2} / \mathrm{H}_{2} \mathrm{O}$ & +0.82 & $200-300\left(\mathrm{O}_{2}\right)$ \\
\hline $\mathrm{O}_{2} / \mathrm{O}_{2}{ }^{\circ}$ & -0.30 & $<0.001\left(\mathrm{O}_{2}{ }^{\circ}\right)$ \\
\hline $\mathrm{O}_{2} \cdot / \mathrm{H}_{2} \mathrm{O}_{2}$ & +0.94 & $1-100\left(\mathrm{H}_{2} \mathrm{O}_{2}\right)$ \\
\hline $\mathrm{H}_{2} \mathrm{O}_{2} / \mathrm{OH}^{\circ}$ & +0.54 & Negligible $\left(\mathrm{OH}^{*}\right.$ ) \\
\hline $\mathrm{OH}^{*} / \mathrm{H}_{2} \mathrm{O}$ & +2.20 & - \\
\hline DHA/ASC & -0.10 & $10,000-20,000$ \\
\hline GSSG/GSH & -0.24 & $2,000-5,000$ \\
\hline $\mathrm{TRX}_{\text {ox }} / \mathrm{TRX}_{\text {red }}$ & -0.33 & $10-100$ \\
\hline NAD $(\mathrm{P}) / \mathrm{NAD}(\mathrm{P}) \mathrm{H}$ & -0.32 & $200-500$ \\
\hline $\mathrm{Fd}_{\mathrm{ox}} / \mathrm{Fd}_{\mathrm{red}}$ & -0.42 & $10-100$ \\
\hline
\end{tabular}

a ASC, ascorbate; DHA, dehydroascorbate; $\mathrm{Fd}$ ox, oxidized ferredoxin; Fd red, reduced ferredoxin; GSH, glutathione; GSSG, glutathione disulfide; TRX ox, oxidized thioredoxin; $\mathrm{TRX}_{\text {red, }}$, reduced thioredoxin.

${ }^{\mathrm{b}}$ High intracellular concentrations of antioxidants prevent indiscriminate oxidation of key cellular components by maintaining oxidant concentrations low. In addition to the abundant pools of ascorbate and glutathione and the battery of peroxide processing enzymes, numerous other compounds can act as chemical antioxidants, including tocopherols, quinones, lipoic acid, carotenoids, and flavonoids.

\section{Acknowledgements}

Research in Professor Shao HB's laboratory is jointly supported by National Science\&Technology Supporting Plan of China (2006BAC01A13) (to Lu ZH), National Natural Science Foundation of China (30770412) (to $\mathrm{Lu} \mathrm{ZH),} 973$ Program of China (2007CB106803), Shao Ming-An's Innovation Team Projects of Education Ministry of China and Northwest A\&F University, and National Natural Science Foundation of China (20772070) (to Kang CM). Thanks are 
also given to three reviewers for their instructive comments and suggestions.

\section{Conflict of interest}

The authors have declared that no conflict of interest exists.

\section{References}

1. Pitzschke A, Hirt H. Mitogen-activated protein kinase and reactive oxygen species signaling in plants. Plant Physiol. 2006;141: 351-356.

2. Chinnusamy V, Schumaker K, Zhu JK. Molecular genetic perspectives on cross-talk and specificity in abiotic stress signaling in plants. J Exp Bot. 2004;55: 225-236.

3. Liu XA, Bush DR. Expression and transcriptional regulation of amino acid transporters in plants. Amino Acids. 2006; (1): 1-8.

4. Zheng W, Wang SY. Antioxidant activity and phenolic compounds in selected herbs. J Agri Food Chem. 2001;49: 5165-5170.

5. Wang WX, Vinocur B, Altman A. Plant responses to drought, salinity and extreme temperatures: towards genetic engineering for stress tolerance. Planta. 2003;218: 1-14.

6. Chaves M. M., Maroco J and Pereira J. Understanding plant responses to drought-from genes to the whole plant. Funct Plant Biol. 2003; 30: 239-264.

7. Stohr C, Stremlau S. Formation and possible roles of nitric oxide in plant roots. J Exp Bot. 2006;57 (3): 463-470.

8. Mullineaux PM, Karpiniski S, Baker NR. Spatial dependence for hydrogen peroxide-directed signaling in light-stressed plants. Plant Physiol. 2006; 141: 346-350.

9. Kwak JM, Nguyen V, Schroeder JI. The role of reactive oxygen species in hormonal responses. Plant Physiol. 2006; 141: 323-329.

10. Halliwell B. Reactive species and antioxidants. Redox biology is fundamental theme of aerobic life. Plant Physiol. 2006;141: 312-322.

11. Couee I, Sulmon C, Gouesbet G. Involvement of soluble sugars in reactive oxygen species balance and responses to oxidative stress in plants. J Exp Bot. 2006; 57 (3): 449-459.

12. Perazzolli M, Romero-Puertas MC, Delledonne M. Modulation of nitric oxide bioactivity by plant haemoglobins. J Exp Bot. 2006; 57 (3): 479-488.

13. Wingler A, Purdy S, MacLean JA. The roles of sugars in integrating environmental signals during the regulation of leaf senescence. J Exp Bot. 2006; 57 (2): 391-399.

14. Fey V, Wagner R, Brautigam K. Photosynthetic redox control of nuclear gene expression. J Exp Bot. 2005; 56 (416): 1491-1498.

15. Scheibe R, Backhausen J, Emmerlich V. Strategies to maintain redox homeostasis during photosynthesis under changing conditions. J Exp Bot. 2005;56 (416): 1481-1489.

16. Coupe SA, Palmer BG, Lake JA. Systemic signaling of environmental cues in Arabidopsis leaves. J Exp Bot. 2006;57 (2): 329-341.

17. Geigenberger P, Kolbe A, Tiessn A. Redox regulation of carbon storage and partitioning in response to light and sugars. J Exp Bot. 2005; 56 (416): 1469-1479.

18. Buchanan BB, Luan S. Redox regulation in the chloroplast thylakoid lumen: a new frontier in photosynthesis research. J Exp Bot. 2005; 56 (416): 1439-1447.

19. Kwon SJ, Choi EY, Choi YJ. Proteomics studies of post-translational modifications in plants. J Exp Bot. 2006;57 (7): 1547-1551.

20. Mur LAJ, Tim L, Carver W. No way to live: The various roles of nitric oxide in plant-pathogen interactions. J Exp Bot. 2006; 57 (3): 489-505.

21. Grun S, Lindermayr C, Sell S. Nitric oxide and gene regulation in plants. J Exp Bot. 2006; 57 (3): 507-516.

22. Crawford NM. Mechanisms for nitric oxide synthesis in plants. J Exp Bot. 2006;57 (3): 471-478.
23. Delledome M, Polverari A, Murgia I. The functions of nitric oxide-mediated signaling and changes in gene expression during the hypersensitive response. Antioxidants \& Redox Sig. 2003; 5 (1): 33-41.

24. Rouhier N, Vlamis-Gardicas A, Lilling CH. Characerization of redox properties of poplar glutaredoxin. Antioxidants \& Redox Sig. 2003;5 (1): 15-22.

25. Link G. Redox regulation of chloroplast transcription. Antioxidants \& Redox Sig. 2003; 5 (1): 79-87.

26. Schurmann P. Redox signaling in the chloroplast: the ferredoxin/thioredoxin system. Antioxidants \& Redox Sig. 2003; 5 (1): 69-78.

27. Barnes D, Mayfield SP. Redox control of posttranslational process in the chloroplast. Antioxidants \& Redox Sig. 2003;5 (1): 89-94.

28. Terman A, Brunk Uft T. Oxidative stress, accumulation of biological'Garbage', and aging. Antioxidants \& Redox Sig. 2006; 8 (1-2): 197-204.

29. Pfannschmidt T, Schutze K, Fey V, et al. Chloroplast redox control of nuclear gene expression-A new class of plastid signals in interorganellar communication. Antioxidants \& Redox Sig. 2003; 5 (1): 95-101.

30. Pfannschmidt T, Liere K. Redox regulation and modification of proteins controlling chloroplast gene expression. Antioxidants \& Redox Sig. 2005; 7 (5-6): 607-618.

31. Kiffin R, Bandyopadhyay U, Cuervo AM. Oxidative stress and autophagy. Antioxidants \& Redox Sig. 2006; 8 (1-2): 152-162.

32. Ivanov B. Participation of photosynthetic electron transport in production and scavenging of reactive oxygen species. Antioxidants \& Redox Sig. 2003;5 (1): 43-53.

33. Kiddle G, Pastori GM, Bernard S, et al. Effects of leaf ascorbate content on defense and photosynthesis gene expression in Arabidopsis thaliana. Antioxidants \& Redox Sig. 2003; 5 (1): 23-32.

34. Aro E-M, Ohad I. Redox regulation of thylakoid protein phosphorylation. Antioxidants \& Redox Sig. 2003; 5 (1): 56-67.

35. Borland A, Elliott S, Patterson S. Are the metabolic components of crassulacean acid metabolism up-regulated in responses to an increase in oxidative burden? J Exp Bot. 2006; 57 (2): 319-328.

36. Yabuta Y, Maruta T, Yoshimura K. Two distinct redox signaling pathways for cytosolic APX induction under photooxdidative stress. Plant Cell Physiol. 2004;45 (11): 1586-1594.

37. Gapper C, Dolan L. Control of plant development by reactive oxygen species. Plant Physiol. 2006;141: 341-345.

38. Sagi M, Fluhr R. Production of reactive oxygen species by plant NAPDH oxidases. Plant Physiol. 2006;141: 336-340.

39. Rio LA del, Sandalio LM, Corpas FJ, et al. Reactive oxygen species and reactive nitrogen species in peroxisomes. Production, scavenging, and role in cell signaling. Plant Physiol. 2006;141: 330-335.

40. Shcolnick S, Keren N. Metalhomeostasis in cyanobacteria and chloroplasts to. Balancing benefits and risks to the photosynthetic apparatus. Plant Physiol. 2006;141: 805-810.

41. Fath A, Bethke P, Beligni V, Jones R. Active oxygen and cell death in cereal aleurone cells. J of Exp Bot. 2002;53: 1273-1282.

42. Cvetkovska M, Rampitsch C, Bykova N, Xing T. Genomic analysis of MAP kinase cascades in Arabidopsis defense responses. Plant Mol Biol Rep. 2005; 23: 331-343.

43. Wu YS, Tang KX. MAP Kinase cascades responding to environmental stress in plants. Acta Bot Sin. 2004; 46 (2): 127-136.

44. Bechtold U, Karpinski S, Mullineaux PM. The influence of the light environment and photosynthesis on oxidative signaling responses in plant-biotrophic pathogen interactions. Plant Cell and Environ. 2005;28: 1046-1055.

45. Hare PD, Cress WA, Staden JVan. Dissecting the roles of osmolyte accumulation during stress. Plant Cell and Environ. 1998;21: 535-553.

46. Igamberdiev AU, Hill RD. Nitrate, NO and haemoglobin in plant adaptation to hypoxia: an alternative to classic fermentation 
pathways. J of Exp Bot. 2004; 55: 2473-2482.

47. Ledford HK, Niyogi KK. Singlet oxygen and photo-oxidative stress management in plants and algae. Plant Cell and Environ. 2005; 28: 1037-1045.

48. Noctor G. Metabolic signaling in defence and stress: the central roles of soluble redox couples. Plant Cell and Environ. 2006; 29: 409-425.

49. Shao HB, Chu LY. Plant molecular biology in China: Opportunities and Challenges. Plant Mol Biol Rep. 2005; 23: 345-358.

50. Wu G, Wei ZK, Shao HB. The mutual responses of higher plants to environment: physiological and microbiological aspects. Biointerfaces. 2007; 59: 113-119.

51. Shao HB, Chu LY, Wu G, Zhang JH, Lu ZH, Hu YC. Changes of some anti-oxidative physiological indices under soil water deficits among 10 wheat (Triticum aestivum L.) genotypes at tillering stage. Biointerfaces. 2007; 54 (2): 143-149.

52. Shao HB, Chen XY, Chu LY, Zhao XN, Wu G, Yuan YB, Zhao CX, $\mathrm{Hu} \mathrm{ZM}$. Investigation on the relationship of Proline with wheat anti-drought under soil water deficits. Biointerfaces. 2006; 53 (2): 113-119.

53. Shao HB, Guo QJ, Chu LY, Zhao XN, Su ZL, Hu YC, and Cheng JF. Understanding molecular mechanism of higher plant plasticity under abiotic stress. Biointerfaces. 2007; 54 (1): 37-45.

54. Shao HB, Jiang SY, Li FM, Chu LY, Zhao CX, Shao MA, Zhao $\mathrm{XN}$, and Li F. Some advances in plant stress physiology and their implications in the systems biology era. Biointerfaces. 2007; 54 (1): 33-36.

55. Shao HB, Chu LY, Zhao CX, Guo QJ, Liu XA, and Ribaut JM. Plant gene regulatory net work system under abiotic stress. Acta Biol Sezeged. 2006; 50 (1-2): 1-9.

56. Shao HB, Liang ZS, Shao MA, Sun Q, Hu ZM. Investigation on dynamic changes of photosynthetic characteristics of 10 wheat (Triticum aestivum L.) genotypes during two vegetative-growth stages at water deficits. Biointerfaces. 2005; 43 (3-4): 221-227.

57. Shao HB, Liang ZS, Shao MA. Adaptation of higher plants to stresses and stress signal transduction. Acta Ecol Sin. 2005; 25: 1871-1882.

58. Shao HB, Liang ZS and Shao MA. Dynamic changes of anti-oxidative enzymes of 10 wheat genotypes at soil water deficits. Biointerfaces. 2005; 42 (3-4): 187-195.

59. Li JM, Jin H. Regulation of brassinosteroid signaling. Trends in Plant Sci. 2007; 12 (1): 37-41.

60. Pourcel L, Routaboul JM, Cheynier V. Flavonoid oxidation in plants: from biochemical properties to physiological functions. Trends in Plant Sci. 2007;12 (1): 29-36.

61. Foyer $\mathrm{CH}$, Noctor $\mathrm{G}$. Redox sensing and signaling associated with reactive oxygen in chloroplasts, peroxisomes and mitochondria. Physiol Plant. 2003;119: 355-364.

62. Foyer $\mathrm{CH}$, Noctor $\mathrm{G}$. Redox homeostis and antioxidant signaling: A metabolic interface between stress perception and physiological responses. Plant Cell. 2005; 17: 1866-1875.

63. Foyer $\mathrm{CH}$, Noctor $\mathrm{G}$. Oxidant and antioxidant signaling in plants: a re-evaluation of the concept of oxidative stress in a physiological context. Plant Cell and Environ. 2005; 28: 1056-1071.

64. Millar AH, Mittova V, Kiddle G. Control of ascorbate synthesis by respiration and its implications for stress responses. Plant Physiol. 2003; 133: 443-447.

65. Nakashima K, Yamaguchi-Shinozaki K. Regulons involved in osmotic stress-responsive and cold stress-responsive gene expression in plants. Physiol Plant. 2006;126: 62-71.

66. Uemura M, Tominaga Y, Nakagawara C, Shigematsu S, Minami A, Kawamura Y. Responses of the plasma membrane to low temperatures. Physiol Plant. 2006; 126: 81-89. 